\title{
Automated 3D EDS Acquisition for Spatially Resolved Elemental Characterization of Catalyzed $\mathrm{MgH}_{2}$ Nanostructures.
}

\author{
Brian Van Devener ${ }^{1}$, Kevin McIlwrath ${ }^{2}$, Steven Kim ${ }^{3}$, Zhigang Zak Fang ${ }^{4}$, Chengshang Zhou ${ }^{4}$ \\ 1. The University of Utah, Surface Analysis Laboratory, Salt Lake City, UT USA. \\ 2. JEOL USA, Inc., TEM Product Division, Pleasanton, CA USA. \\ 3. AppFive, Tempe, AZ USA. \\ 4. The University of Utah, Department of Metallurgical Engineering, Salt Lake City, UT USA
}

Since the introduction of large area Silicon Drift Detectors (SDD) for EDS, the acquisition time for high resolution EDS maps has decreased by more than an order of magnitude. For example, typical EDS mapping times with good signal to noise for a 256 x 256 pixel map can be now acquired in less than 3 minutes, depending upon the concentration of mapped elements within the sample and incident probe current on the sample. Combined with advances in computer automation of electron microscopes, this has made the acquisition of three-dimensional compositional data via EDS tilt tomography a practical reality.

Here, we present results showing a reconstructed 3D EDS and 3D STEM HAADF data set of a $\mathrm{MgH}_{2}$ nanocrystallite embedded with $\mathrm{V}$, Ti and $\mathrm{Cr}$ which serve as hydrogenation/dehydrogenation catalysts. Figure 1 shows one tilt frame of the 3D reconstructed EDS spectral map animation.

The tomographic data set was acquired using the AppFive Iris software interfaced to a JEOL JEM-2800 $200 \mathrm{kV}$ FE-TEM/STEM equipped with twin $100 \mathrm{~mm}^{2}$ SDD EDS detectors - which have a combined, nominal solid angle of collection of $1.6 \mathrm{sr}$. The sample was tilted through a range of -56 to +64 degrees with $750 \times 708$ pixel maps acquired in 4 degree steps. Total collection time for the EDS spectral images was 30 seconds per frame averaged over 10 frames per tilt step which gave a data collection time of $\sim 2.5$ hours. Acquisition time for the entire experiment, including auto-focus, tracking and other parameters, required 3 hours and 17 minutes. Alignment, reconstruction and visualization of the image/elemental stacks was accomplished using Composer and Visualizer Kai post processing software developed by Systems In Frontiers which has been optimized for batch processing of elemental tilt series data.

Proper selection of a sample holder that offers high tilt and a good line of site towards both EDS detectors is critical for this type of experiment. In this case, the JEOL half grid high tilt retainer (HTR) was chosen, see Figure 2. With the pole piece gap of the JEOL 2800, this holder has a tilt range of +/$80^{\circ}$.

Nanostructured materials with catalytically active sites have long been recognized as a class of materials with inherent properties which make them useful as model systems to be studied using 3D tomographic, characterization methods [1]. For these materials, an understanding of nanoparticulate shape and distribution of catalytically active elements is critical in developing models to better understand structure/material relationships. The challenges in applying tomographic EDS analysis to such materials are well known: the limited collection efficiency of X-Ray counts with earlier EDS detector designs coupled with the large number of tilt slices needed to reconstruct the 3D volumetric data set, typically have required extremely long data acquisition times which are unfavourable in terms of beam induced 
damage for low $\mathrm{Z}$ containing and/or non-thermally conductive materials. EDS collection efficiency such as that found on the JEOL 2800, coupled with an automated tilt software control system, such as the AppFive system used in this study, are breaking down these long standing barriers.

Magnesium hydride is well known as a possible material for hydrogen storage and thermal energy storage given its low cost, high theoretical hydrogen capacity, good reversibility, and high energy density in terms of magnesium and hydrogen reactions [2,3]. Unfortunately, the kinetics of the dehydrogenation - hydrogenation reaction of $\mathrm{MgH}_{2}$ are unfavourable. Research has shown great enhancement of the kinetic rate of the hydrogenation/dehydrogenation cycle, by reducing the $\mathrm{Mg} / \mathrm{MgH} 2$ to the nanoscale, and by doping the $\mathrm{MgH}_{2}$ with catalysts - namely transition metals such as $\mathrm{V}, \mathrm{Cr}$, and $\mathrm{Ti}[4,5]$.

In order to better understand the transition metal catalyst effects on the hydrogenation/dehydrogenation of $\mathrm{MgH}_{2}$ it is critical to understand the kinetics in terms of the microstructural evolution of $\mathrm{Mg}$ and $\mathrm{MgH}_{2}$ as well as in the spatial distribution of catalytically active metals throughout the mixed material nano-clusters. A recent two part study investigated this relationship [6]. Here, it was found through a combination of: XRD, SEM, TEM, XPS and traditional 2D STEM/EDS spectral imaging measurements, that by comparing before and after hydrogen cycled $\mathrm{MgH}_{2}-\mathrm{VTiCr}$ nano clusters, the distribution of the transition metal catalysts changed from being distributed throughout the aggregates to predominantly segregated on the surface, for before and after cycled samples respectively. The same conclusion is elegantly demonstrated by the tomographic data set in this work.

\section{References:}

[1] M.S. Moreno, et al, Micron 37 (2006) 52-56

[2] L. Schlapbach, A. Zuttel, Nature 414 (2001) 353-358

[3] Z.Z. Fang, et al, J. Alloys Compd. 645 (2015) S184

[4] Y.J. Choi, et al, J. Power Sources 180 (2008) 491-497

[5] C. Zhou, et al, J. Power Sources 278 (2015) 38-42

[6] C. Zhou, et al, J. Phys. Chem. C 119 (2015) 22261-22271, 22272-22280

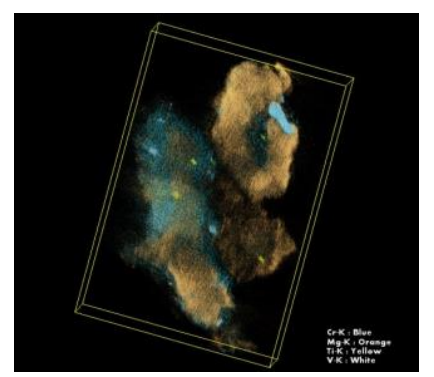

Figure 1. 3D-EDX Data Visualization of $\mathrm{MgH}_{2}-\mathrm{VTiCr}$ nano structure

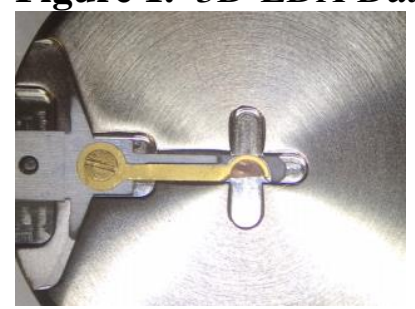

Figure 2. JEOL half grid high tilt retainer TEM sample holder 